\title{
Short-chain methotrexate polyglutamate MTXPG2 as outcome parameter in rheumatoid arthritis
}

\author{
Eva-Luise Hobl ${ }^{*}$, Bernd Jilma', Ludwig Erlacher ${ }^{2}$, Robert Mader ${ }^{3}$ \\ From 16th Scientific Symposium of the Austrian Pharmacological Society (APHAR) \\ Vienna, Austria. 25-27 November 2010
}

\section{Background}

Due to its positive benefit-risk-ratio, methotrexate (MTX) is a first-line therapy in the treatment of rheumatoid arthritis (RA). Although MTX is very effective, the large interpatient variability in drug response is a major drawback in clinical practice. Erythrocyte methotrexate polyglutamates (MTXPG1-7) - intracellular storage products of methotrexate - are supposed to correlate with clinical efficacy. In particular, long-chain polyglutamates (MTXPG4-5) are strongly retained in the cells and are therefore discussed to be potential markers for clinical response in rheumatoid arthritis. We hypothesized that concentrations of methotrexate polyglutamates could correlate with clinical response parameters in rheumatoid arthritis.

\section{Methods}

A randomized, double-blinded controlled clinical trial including nineteen patients was performed according to GCP (Good Clinical Practice) guidelines and ethical principles that have their origin in the Declaration of Helsinki. Patients received a dose of $25 \mathrm{mg}$ methotrexate per week administered orally. Laboratory testing and pharmacokinetics were performed at week 1 , week 5 , week 10 and at the end of study in week 16 . The primary outcome parameter for clinical evaluation was DAS-28 (Disease Activity Score in 28 joints). DAS-28 calculation incorporates clinical parameters (ESR, swollen and tender joints) as well as subjective parameters (VAS pain). For measurement of methotrexate polyglutamates in erythrocytes, we used an HPLC (High Pressure Liquid Chromatography) method with post-column photooxidation followed by fluorimetric detection.

\footnotetext{
* Correspondence: eva-luise.hobl@meduniwien.ac.at

'Department of Clinical Pharmacology, Medical University of Vienna, 1090

Vienna, Austria

Full list of author information is available at the end of the article
}

\section{Results}

Correlation of pharmacokinetics and clinical parameters using Spearman's correlation coefficient showed a statistically significant positive correlation of $\mathrm{C}_{\max }$ values of MTXPG2 and improvement in DAS-28 $(+0.518, \mathrm{p}=$ 0.023 ) at week 5 . This correlation could indicate that erythrocyte concentrations of MTXPG2 are relevant for clinical response and potentially for drug monitoring. Up to now, only the accumulation of long-chain polyglutamates was linked to a good MTX response.

\section{Conclusions}

In particular, MTXPG2 seems to be an indicator for clinical response and may serve as a marker for drug monitoring. These seminal data should stipulate further trials to define the potential role of measuring erythrocyte concentrations of MTX for clinical prediction of MTX response.

\section{Author details \\ 'Department of Clinical Pharmacology, Medical University of Vienna, 1090 Vienna, Austria. ${ }^{2}$ Department of Rheumatology, Kaiser-Franz-Josef-Spital, 1100 Vienna, Austria. ${ }^{3}$ Department of Internal Medicine I, Medical University of Vienna, 1090 Vienna, Austria.}

Published: 16 November 2010

doi:10.1186/1471-2210-10-S1-A35

Cite this article as: Hobl et al:: Short-chain methotrexate polyglutamate

MTXPG2 as outcome parameter in rheumatoid arthritis. BMC

Pharmacology 2010 10(Suppl 1):A35. 